\title{
Le analisi costo della malattia e costo efficacia in farmacoeconomia. Ambiti di applicabilità, problemi, prospettive.
}

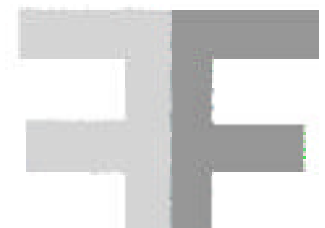

METODI

di Piervincenzo Bondonio a

\begin{abstract}
Aim of the paper is to compare and evaluate some of the main features of two different tecniques of economic analyses: cost of illness e and cost-effectiveness. The former is not so widely used in pharmacoeconomics, while the latter is dominant. Although their theory has recently much progressed, their practice is still hindered by some unresolved questions, to which the paper also addressess.
\end{abstract}

Farmeconomia e percorsi terapeutici 2000; 1 (1): 9-18

\section{INTRODUZIONE}

Il titolo stesso di questo scritto, assegnatomi dagli organizzatori del Convegno, con l'accostare 1'analisi costo della malattia (Cost of illness, COI) all' analisi costo efficacia (Cost Effectiveness Analysis, CEA) mette in evidenza, senza equivoci, che la sua funzione è soprattutto didascalica.

I due tipi di analisi seguono logiche e perseguono obiettivi che sono largamente distinti: metterli in luce, delinearne il rispettivo ambito di applicabilità e di utilizzabilità, rimarcando le eventuali zone di convergenza o complementarietà costituisce pertanto l'obiettivo primario di questo scritto.

Il lavoro non presenta studi originali, in nessuno dei due tipi di analisi ma, utilizzando una piccola parte di un'ormai vastissima letteratura, prova a interpretare le relazioni possibili tra i due filoni di analisi (il primo, per la verità, assai meno praticato del secondo), focalizzando l'attenzione sugli aspetti verso i quali si è coagulato un vasto assenso e distinguendoli dalle aree tuttora aperte alla discussione e al dissenso (per fortuna, ancora numerose).

Poichè altre relazioni esplorano in profondità specifici aspetti delle analisi economiche (della CEA, essenzialmente), l'attenzione sarà qui concentrata su alcuni aspetti metodologici generali e sul tema dell'utilizzabilità delle analisi.

In generale si possono apprezzare le differenze tra COI e CEA considerando che la CEA trovi una sua chiara collocazione nel noto schema classificatorio delle tecniche di analisi economica in Sanità, costruito tenendo conto del diverso grado di completezza dell'analisi (si a università di Torino valutano i costi, i benefici dei trattamenti o e Cresa. Relazione entrambi?) e del fatto che l'analisi sia appli- presentata $a l 1^{\circ}$ cata o meno a più programmi alternativi di Convegno nazionale intervento (vedi Tabella 1 (9), (6)), mentre la COI invece non vi compare.

La CEA è una delle tecniche di valutazione tra alternative terapeutiche nella quale sono presi in considerazione, contemporaneamente $\mathrm{i}$ costi delle terapie e $\mathrm{i}$ benefici derivanti dalla loro adozione (questi ultimi non sotto forma monetaria, ma di risultato in termini di salute). L'analisi, pur di tipo positivo-descrittivo, ha immediato utilizzo in termini normativi (cioé per aiutare a effettuare scelte tra terapie alternative), a livelli che possono spaziare dal micro (singole unità operative), al meso (ad esempio un insieme di ospedali), al macro (un sistema sanitario).

La COI, invece, è una tecnica economica mediante la quale si calcolano i costi (tutti o alcuni) che una determinata società sopporta per effetto dell'incidenza di una data patologia. Lo scopo è di rappresentare la perdita di ricchezza che, a un livello territoriale sufficientemente aggregato (quello al quale sono disponibili dati epidemiologici significativi), la società nel suo insieme subisce come conseguenza della patologia, a prescindere dalla possibilità e dalle modalità di affrontare con successo la patologia stessa. Si tratta pertanto di un'analisi positiva (cioé con scopi descrittivi o predittivi), condotta a livello macro e focalizzata sui costi ascrivibili alle malattie e non sulle terapie per affrontarle. 
LE ANALISI COSTO DELLA MALA TTIA: UTILI STRUMENTI CONOSCITIVI, MA DI UTILIZZO PROBLEMATICO

\section{Uno scenario}

Non diversamente da qualunque altro feNota i

Ad esempio, fenomeni naturali come terremoti e innondazioni, o sociali come l'analfabetismo, o economici come l'impatto del progresso tecnologico di altri sistemi economici o la riduzione della domanda mondiale di un bene prodotto da un dato Paese.

\section{Nota ii}

Più frequenti sono forse $i$ casi in cui le società si muovono soprattutto sull'onda di fatti emotivi, che colpiscono la fantasia popolare e inducono a ritenere che "occorra fare qualcosa" per porre rimedio al fenomeno negativo, inteso come manifestazione ingiusta di una natura matrigna (e non, magari, di umana insipienza passata, quale invece spesso è).

\section{Tabella 1}

Tipi di tecniche di analisi farmacoeconomica (9) problema, prima che si conoscano i costi e le probabilità di successo.

\section{Metodo, contenuti e risultati della COI: un esempio}

Per illustrare i contenuti della COI si fa inizialmente riferimento a uno studio elaborato dal Cresa (10), relativo a una malattia a crescente impatto nella società italiana contemporanea, caratterizzata dal progressivo invecchiamento della sua popolazione: la demenza senile, una delle patologie degenerative più diffuse del sistema nervoso centrale.

Si trattava in primo luogo di stimare l'impatto quantitativo della patologia nella società italiana. Questa malattia interessa una quota rilevante della popolazione anziana (dal 6 al $10 \%$ degli ultra65enni) e gli studi epidemiologici indicano una crescita della diffusione all'aumentare dell'età. Tale crescita è stata stimata in un raddoppio della prevalenza, a partire dai 60 anni e fino ai 95, passando da una classe di cinque anni alla successiva.

In assenza di dati statistici di base affidabili (non esisteva, per molte ragioni, un'anagrafe delle persone colpite dalla patologia) è stato necessario effettuare un'accurata analisi dei risultati delle indagini epidemiologiche condotte in Italia sulla demenza, per individuare quelli ritenuti più persuasivi (per metodo di indagine e per contesto di svolgimento) e generalizzarne poi i risultati all'intera società italiana. Si è così pervenuti a una stima di incidenza complessiva di circa 650.000 casi (350.000 dei quali classificabili come lievi).

Successivamente si è valutato, a livello macroeconomico (in questo caso italiano) e con

\begin{tabular}{|c|c|c|}
\hline $\begin{array}{c}\text { NUMERO DI } \\
\text { ALTERNATIVE } \\
\text { CONSIDERATE } \\
\end{array}$ & $\begin{array}{l}\text { PARAMETRI } \\
\text { ANALIZZATI }\end{array}$ & $\begin{array}{l}\text { DENOMINAZIONE } \\
\text { DELLA TECNICA }\end{array}$ \\
\hline \multicolumn{3}{|l|}{ Analisi parziali } \\
\hline considera una sola terapia & analizza solo i costi & descrizione dei costi \\
\hline considera una sola terapia & analizza solo gli effetti & descrizione degli effetti \\
\hline considera una sola terapia & analizza sia costi che effetti & $\begin{array}{l}\text { valutazione dei costi e dei } \\
\text { benefici }\end{array}$ \\
\hline $\begin{array}{l}\text { confronta due o più terapie } \\
\text { alternative }\end{array}$ & analizza solo i costi & analisi comparativa dei costi \\
\hline $\begin{array}{l}\text { confronta due o più terapie } \\
\text { alternative }\end{array}$ & analizza solo gli effetti & $\begin{array}{l}\text { analisi comparativa di } \\
\text { efficacia o di efficienza }\end{array}$ \\
\hline \multicolumn{3}{|l|}{ Analisi complete } \\
\hline $\begin{array}{l}\text { confronta due o più terapie } \\
\text { alternative }\end{array}$ & $\begin{array}{l}\text { analizza sia i costi sia gli } \\
\text { effetti }\end{array}$ & \begin{tabular}{|l} 
- minimizzazione dei costi \\
- analisi costi-efficacia \\
- analisi costi-efficienza \\
- analisi costi-utilità \\
- analisi costi-benefici
\end{tabular} \\
\hline
\end{tabular}


riferimento alle principali categorie di costo, l'ammontare delle risorse impiegate dall'intera collettività nazionale per la diagnosi, il trattamento e l'assistenza dei dementi; in un'accezione più vasta, potevano essere anche presi in considerazione $\mathrm{i}$ costi indiretti e quelli intangibili.

I problemi metodologici da risolvere, in questo caso, non sono diversi da quelli che devono essere affrontati nella CEA (e nelle altre forme di valutazione economica): si tratta di decidere, infatti, quali costi prendere in considerazione e con quale metodologia valutarli.

Con riferimento a un'ampia gamma di costi che possono essere considerati in una tipica COI, lo studio qui ricordato ha effettuato le sue scelte, giustificandole con ragioni di carattere in parte generale e teorico, in parte pratico.

In linea di principio, il punto di vista considerato (che accomuna la COI alla CEA) è quello collettivo o societario: i costi presi in considerazione sono quelli sostenuti da qua- lunque parte, non importa quale (l'organizzazione sanitaria pubblica, il paziente e la sua famiglia - i caregiver -, la collettività più ampia). Ciò implica, da un lato, un'estensione del campo di indagine, la cui attuazione può presentare tuttavia difficoltà pratiche, che possono suggerire un successivo restringimento del campo di indagine nell'effettuazione dei calcoli; d'altro lato, invece, comporta l'eliminazione dai costi considerati di tutto ciò che presenta caratteristiche di spesa di trasferimento (che grava su alcuni soggetti, ma ne avvantaggia altri). Nel caso dello studio qui considerato non tutti i costi potenzialmente rilevanti sono stati valutati, per ragioni legate alla disponibilità di informazioni adeguate e in qualche caso, per la presumibile scarsa rilevanza di alcune voci. Di fatto, lo studio è pervenuto a una prima stima dei costi diretti sanitari, distinguendo tra spese: per l'attività diagnostica; per il trattamento farmacologico (trattamento dei disturbi cognitivi legati all'invecchiamento del sistema nervoso centrale); per i ricoveri ospedalieri (ordinari e in regime diur-

\begin{tabular}{|l|}
\hline COSTI DIRETTI: \\
Sanitari: \\
accertamenti diagnostici \\
visite mediche trattamenti farmacologici \\
assistenza infermieristica \\
accessi a day hospital \\
utilizzo di centri diurni \\
ricoveri ospedalieri \\
prestazioni di riabilitazione \\
servizi preventivi e di profilassi \\
protesi e ausili \\
ricoveri in residenze sanitarie \\
Non sanitari: \\
trasporti (per accesso ai servizi sanitari) \\
collaborazioni domestiche \\
assistenza e sorveglianza \\
modificazioni dell'abitazione e dell'alimentazione del paziente \\
protesi e ausili \\
servizi sociali \\
ricoveri in residenze assistenziali \\
\hline COSTI INDIRETTI: \\
tempo sottratto ad attività lavorativa (pazienti e loro familiari) \\
tempo sottratto ad attività lavorativa (pazienti e loro familiari) \\
perdita di produttività (pazienti e loro familiari) \\
\hline COSTI INTANGIBILI: \\
sofferenza dovuta ad ansietà, dolore, inabilità, isolamento affettivo e sociale \\
conflitti familiari, modificazione degli stili di vita, ecc. \\
\hline
\end{tabular}

Tabella 2

Tipologia di costi considerabilinella COI della demenza senile. (10) 
no; per i ricoveri in strutture residenziali (pubbliche e private); per gli ausili per incontinenti. Tra i costi diretti non sanitari sono poi stati valutati gli assegni di accompagnamento a carico del Ministero dell'Interno. L'ultima voce di costo considerata, che assorbe da sola quasi il $70 \%$ del COI totale stimato, è attribuita a una pur prudenziale valutazione del tempo dedicato all'assistenza dai caregiver, normalmente - in Italia - familiari degli anziani malati.

Tra i problemi insiti nella valutazione dei costi, quello che merita particolare attenzione è la scelta del percorso diagnostico e terapeutico.

Mentre nella CEA la contrapposizione di due o più percorsi costituisce la ragione d'essere dell'analisi (che intende, appunto, confrontare i costi - ma anche i risultati - differenziali di due o più percorsi, alla ricerca di quello "più costo-efficace"), nella COI la valutazione può prendere invece in considerazione, alternativamente, il percorso più frequentemente utilizzato (o un mix ponderato tra i percorsi utilizzati, se l'informazione per effettuare la ponderazione è nota), oppure, se è stato selezionato sulla base dei risultati di CEA, solamente il percorso più costo-efficace. Nel primo caso la COI stimerà i costi diretti di malattia conseguenti all'adozione dei percorsi diagnostico-terapeutici prevalenti, anche se economicamente non ottimali; nella seconda, quelli che scaturiscono dalle scelte diagnostico-terapeutiche economicamente più efficaci.

Nella tabella 3 sono riportati i risultati finali dello studio, ottenuti con riferimento agli aggregati di costo annuo per i quali è stata effettuata la stima, che non comprende né una valorizzazione della perdita di produttività (da parte dei pazienti e dei loro familiari), né dei costi intangibili; le due serie di valori rappresentano la forbice all'interno della quale si è ritenuto possa ricadere il valore "vero".
Sulla base di tali valutazioni lo studio perviene alla stima di un costo annuo per paziente che si colloca tra gli 11 e i 15 mila dollari, dei quali una cifra compresa tra i 6.500 e i 9.700 dollari sono a carico delle famiglie.

L'utilizzo e la diffusione della COI: prospettive e problemi

L'utilità potenziale della COI, con una percezione dei problemi che il suo utilizzo comporta, sono bene evidenziati dagli studi che effettuano confronti diretti tra i costi annui mediamente sostenuti per ogni paziente affetto da diverse patologie.

Ad esempio la Tabella 4, ripresa da uno studio di Greenberg (12), compara il costo annuale negli USA di alcune malattie di rilevante peso sociale, mettendo in relazione tali costi con la prevalenza delle patologie considerate e con la sopravvivenza, la diagnosticabilità e la possibilità terapeutica.

Gli autori, nel nostro caso medici psichiatri, hanno buon gioco nel dimostrare che, sulla base dei risultati COI integrati con altre informazioni rilevanti, "vale la pena" investire nella diagnosi e nel trattamento della depressione. Questa patologia coinvolge negli Stati Uniti 15 milioni di persone, per lo più giovani e inseriti in attività produttive, ed è una malattia:

? con elevata sopravvivenza e curabile con ottimo successo;

? che assorbe una quantità di risorse pari a quella assorbita dalle malattie coronariche, nonostante esse interessino meno della metà dei suoi pazienti;

? con un costo sociale pari al $43 \%$ del cancro e al $67 \%$ dell'AIDS, i cui malati sono invece del 60 e del 98,7\% meno numerosi.

In questo caso, come in molti altri, lo studio ha lo scopo di richiamare l'attenzione su un problema (qui la diagnosi precoce e la cura delle forme depressive) al quale "la società"
Tabella 3

Costo annuo della demenza senile in Italia (valori in milioni di dollari USA 1993, al tasso di cambio di lire 1650 per 1 dollaro USA; valori arrondati alla decina piùvicina)(10)

\begin{tabular}{|l||r|r|}
\hline COSTI DIRETTI SANITARI & 350 & 635 \\
accertamenti diagnostici & 150 & 210 \\
trattamenti farmacologici & 20 & 30 \\
ricoveri ospedalieri & 1.210 & 1.815 \\
residenze assistenziali per anziani & 300 & 420 \\
ausilii per incontinenti & & \\
COSTI DIRETTI NON SANITARI & 240 & 360 \\
assegni di accompagnamento & $\mathbf{2 . 2 7 0}$ & $\mathbf{3 . 4 7 0}$ \\
Totale costi diretti & & \\
\hline COSTI INDIRETTI & 4.850 & 5.450 \\
$\quad$ assistenza dei familiari & $\mathbf{7 . 1 2 0}$ & $\mathbf{9 . 9 2 0}$ \\
\hline \hline COSTI TOTALI & & \\
\hline
\end{tabular}


dedica insufficiente attenzione (in questo caso rispetto a quella dedicata ad altre patologie, che hanno esiti più gravi - mortali - ma riguardano anche un numero minore, anche molto minore, di persone e, soprattutto, per le quali sussistono terapie meno efficaci e più costose).

Ci si può chiedere chi siano i veri destinatari di studi di questa natura. In questo caso lo studio è stato pubblicato su una rivista scientifica disciplinare (il Journal of Clinical Psychiatry) e quindi si deve ritenere che i primi destinatari siano i suoi abituali lettori. Tuttavia è evidente che esso si rivolge a un pubblico ben più vasto: a chi può, con il suo comportamento e la sua capacità di influenza, contribuire a fare cambiare le decisioni collettive, nella direzione auspicata, dedicando più risorse alla diagnosi precoce e alla cura della depressione.

Questa banale constatazione porta a qualche riflessione aggiuntiva sul ruolo delle analisi COI, su chi possa (debba?) realizzarle e sui suoi destinatari.

La realizzazione di una buona analisi COI deve affrontare le stesse sfide insite in altri tipi di studi economici, come le analisi costi-benefici, costi-efficacia e costi-utilità. In particolare, deve individuare gli elementi di costo più rilevanti ai fini della valutazione economica, precisarne i metodi, identificarne limiti e potenzialità. Inoltre è necessario che disponga di solide conoscenze epidemiologiche, per stimare correttamente la prevalenza delle patologie nel contesto in cui esse si manifestano, e - come si è già accennato - conoscere il percorso diagnostico e terapeutico utilizza- to per affrontarle. In altre parole, è evidente che le analisi COI richiedono, come e ancora più che le altre valutazioni economiche, apporti pluridisciplinari: accanto all'economista sanitario e allo statistico dovranno operare il demografo e l'epidemiologo, il clinico e il farmacologo clinico.

Probabilmene in ragione della loro maggiore complessità, o della minore "appropriabilità" dei risultati cui possono dare luogo, le analisi COI sono meno praticate, ad esem-

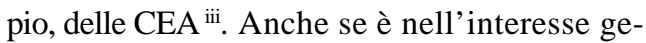
nerale che buone analisi COI siano periodicamente effettuate, almeno con riferimento alle patologie di maggior impatto sociale. Tuttavia, alla definizione di regole da seguire nell'effettuare "buone" analisi COI non è stata dedicata analoga attenzione di quella impiegata a costruire, ad esempio, linee guida per le analisi farmacoeconomiche di tipo CEA.

Ciò non significa però che regole non esistano. Esse sono infatti ricavabili dai metodi che governano la ricerca scientifica nei rispettivi campi disciplinari che convergono negli studi COI.

Chi può effettuare le analisi COI? La risposta, oggi, sembra univoca: chiunque lo voglia, naturalmente, e qualunque sia l'interesse che lo spinge a farlo. Sarà responsabilità della comunità scientifica valutare e validare le analisi effettuate - i cui realizzatori mettono peraltro in gioco la propria reputazione - alla luce dei criteri e dei metodi sviluppati negli ambiti disciplinari.

Può tuttavia accadere (e mi pare che, in Italia, si sia prossimi a una situazione di questo genere) che le forze di mercato (anche del

\begin{tabular}{|c|c|c|c|c|}
\hline & Depressione & $\begin{array}{c}\text { Malattia } \\
\text { coronarica }\end{array}$ & Cancro & AIDS \\
\hline Prevalenza (milioni) & 15 & 7 & $\mid 6$ & 0,2 \\
\hline Distribuzione per età & $\begin{array}{l}70 \% \\
(18-45 \text { a. })\end{array}$ & $\begin{array}{l}95 \% \\
(>44 \text { a.) }\end{array}$ & $\begin{array}{l}80 \% \\
(20-40 \text { a. })\end{array}$ & \\
\hline $\begin{array}{l}\text { Distr. per sesso: } \\
\text { uomini } \\
\text { donne }\end{array}$ & $\begin{array}{l}29 \% \\
71 \%\end{array}$ & $\begin{array}{l}56 \% \\
44 \%\end{array}$ & \begin{tabular}{||l}
$50 \%$ \\
$50 \%$
\end{tabular} & $\begin{array}{l}89 \% \\
11 \%\end{array}$ \\
\hline Sopravvivenza a 5 anni & Alta & Varia & Varia & Bassa \\
\hline Diagnosticabilità & Bassa & Alta & Alta & Alta \\
\hline Possibilità terapeutica & Alta & Varia & Varia & Bassa \\
\hline $\begin{array}{l}\text { Costo annuo totale } \\
\text { (miliardi US \$) }\end{array}$ & 44 & 43 & |104 & 66 \\
\hline Costo annuo/paziente (US \$) & 2.933 & $\mid 6.143$ & $\mid 17.333$ & 330.000 \\
\hline $\begin{array}{l}\text { Rapporto del costo } \\
\text { annuo/paziente rispetto a } \\
\text { depressione }\end{array}$ & 1 & 2,09 & $\mid 5,91$ & 112,51 \\
\hline
\end{tabular}

\section{Nota iii}

Mentre annualmente sono pubblicate, su riviste scientifiche mediche, oltre un centinaio di analisi di tipo CEA (ma molto poche su riviste propriamente economiche), una ricerca condotta qualche tempo fa su MedLine evidenziava la presenza complessiva, nella banca dati interrogata, di soli 236 lavori di tipo COI (19).

Tabella 4

COI e prevalenza di alcune patologia di rilevante interesse sociale caratterizzate da diversa distribuzione demografica, sopravvivenza, diagnosticabilitàe possibilità terapeutiche (12) 
mercato della ricerca universitaria) non producano, in un dato momento, un flusso di analisi giudicato adeguato, per quantità e qualità. Avvertire il problema è comunque la premessa per avviarlo a soluzione: se la collettività nazionale avesse coscienza di un tale problema, le sarebbe possibile intervenire per risolverlo, attraverso i normali canali di incentivazione della ricerca e attivando meccanismi di competizione tra ricercatori e centri di ricerca, senza escludere di assegnare un ruolo diretto - ma mai monopolistico - a organismi di ricerca pubblici (quali, ad esempio, l'Istituto Superiore di Sanità o il CNR).

LE ANALISI COSTO-EFFICACIA: QUALE AUSILIO PER LE DECISIONI E A QUALE LIVELLO?

\section{Cos'è la CEA e come può essere usata}

Secondo una definizione molto generale, la CEA "is a method designed to assess the comparative impact of expenditures on different health interventions" (17). Essa è basata sulla premessa che "for any given level of resources available, society...wishes to maximize the total aggregate health benefits conferred" (18).

Nella CEA ruolo centrale è giocato dal rapporto costo/efficacia (C/E): nel confronto tra due o più alternative (ad esempio, tra un nuovo farmaco e il farmaco abitualmente usato, o un placebo, o il migliore farmaco prima disponibile), il rapporto costo/efficacia è calcolato come rapporto tra la differenza nei loro costi e la differenza nella loro efficacia.

Il rapporto $\mathrm{C} / \mathrm{E}$ è quindi interpretabile

Nota iv

Questa visione, propria di filosofi sociali come Rawls (14), è associata all'idea che sia possibile prendere decisioni nel pubblico interesse.

\section{Nota $\mathbf{y}$}

Il ragionamento si applica anche alle misura alternative, o complementari al QALY, che sono state sviluppate e sono occasionalmente utilizzate nella CEA (quali, ad esempio: healthy years equivalents, saved young life equivalents). rapporto $\mathrm{C} / \mathrm{E}$ inferiori o eguali a un certo valore sarebbe economicamente ottimale, nel senso che: 1) gli interventi così realizzati renderebbero massimo l'effetto sulla salute conseguibile con le risorse disponibili, e 2) il risultato totale in termini di salute sarebbe conseguito con il minimo costo possibile (17).

Una questione preliminare: quale prospettiva per l'analisi? Una soluzione, con qualche dubbio

A risultati di questo genere la CEA non perviene tuttavia in modo né facile né indolore. Per influenzare le decisioni sociali la CEA deve possedere caratteristiche che rendono impegnativa la sua pratica e, in alcuni casi, ne mettono in discussione la teoria. Di seguito sono discussi alcuni aspetti del problema.

Un punto preliminare riguarda la scelta della prospettiva dalla quale le valutazioni devono essere condotte. Come è noto, è opinione prevalente che la CEA assuma il punto di vista societario: l'analista deve cioè prendere in considerazione tutti i costi (da chiunque sopportati) e tutti i risultati sulla salute (da chiunque sperimentati, positivi o negativi che siano). Questa scelta può comportare che alcune decisioni siano in contrasto con gli interessi di persone alle quali, sulla base di considerazioni in parte derivanti da CEA, tali programmi siano stati negati (ad esempio, l'accesso a un trapianto renale di persone così anziane $\mathrm{o}$ in stato di salute così precario che il trapianto migliorerebbe di poco le loro aspettative di vita). La razionalità e l'accettabilità da parte di tutti di una scelta siffatta si fonda su una visione contrattualista del patto sociale ${ }^{\mathrm{iv}}$. Ognuno, ignorando quale destino gli riserverà la vita, razionalmente desidera che le decisioni sull'allocazione delle risorse siano effettuate in modo tale da assicurargli un'adeguata assistenza qualunque problema di salute egli possa in futuro incontrare. In questo ambito, solo la prospettiva societaria è in grado di garantire che non sia calcolato come vantaggio per qualcuno quello che è un danno per un altro. Naturalmente è legittimo effettuare analisi $\mathrm{C} / \mathrm{E}$ adottando punti di vista diversi e più ristretti, ad esempio, quello di specifiche categorie di persone (i dializzati, le persone in attesa di trapianto, eccetera) o di una specifica organizzazione sanitaria, anche generale, come il SSN italiano. Non di meno, in tali casi, la valutazione condotta dalla prospettiva societaria sarà utile, in quanto permetterà di disporre di un termine di confronto per valutare le analisi particolaristiche.

La priorità accordata alla prospettiva societaria ha evidenti implicazioni di principio su quali costi e quali risultati la CEA deb- 
ba valutare e come essi debbano essere misurati. Nel mondo reale, tuttavia, la prospettiva societaria può implicare considerazioni che non sono di fatto riflesse (e in qualche caso, neppure lo potrebbero) nelle misure di costo e di risultato della CEA (17).

Con riferimento ai risultati, la più classica misura "grezza" di outcome è l'anno di vita guadagnato o salvato. Esso, tuttavia, non è necessariamente l'unico risultato di un programma sanitario di successo, che potrebbe annoverare tra i suoi effetti positivi anche il sollievo dal dolore, il miglioramento delle prestazioni, una migliore informazione, il fatto di convogliare cura e attenzioni altrui. Il QALY $^{\mathrm{v}}$ e le altre misure di outcome sviluppate cercano di tenere conto dei fattori qualitativi che connotano una maggiore durata della vita, senza tuttavia riuscirci del tutto.

Inoltre si pone il "problema dell'aggregazione", che considera quale sia il punto di equilibrio tra la somma di modesti benefici riguardanti molte persone e la somma di elevati benefici per un ristretto numero di persone: supponendo pari numero di QALYs, la valutazione sociale è davvero la stessa? Dobbiamo riconoscere che le società contemporanee si arrovellano su problemi di giustizia distributiva, alcuni dei quali sono lontani dal ricevere soluzioni convincenti e durature, quindi il QALY e gli altri simili indicatori quantitativi, che potrebbero essere misure soddisfacenti di risultato dal punto di vista societario solo se si fosse in grado di ponderarli adeguatamente, mancando tale possibilità non posseggono questa caratteristica $(5)^{\mathrm{vi}}$. E ancora: non tutti i valori pubblici di rilievo possono essere incorporati nella CEA: non lo è, ad esempio, il diritto alla privacy che impedisce, al di fuori di specifiche e limitate circostanze, la diagnosi obbligatoria dell' HIV, anche in presenza di trattamenti in grado di prolungare la vita nei confronti dei pazient diagnosticati precocemente. In tali circostanze, "le misure di risultato sanitario utilizzate nella CEA sono destinate a rimanere rappresentazioni parziali degli obiettivi e dei valori societari” (17).

Con riferimento ai costi, la CEA dovrebbe prendere in considerazione anche il costo opportunità delle risorse impiegate dal paziente e da chi gli fornisce assistenza gratuita (figurano entrambi tra i costi indiretti della tab. 1). Al di là delle difficoltà di calcolo, che possono essere risolte con riferimento a valori salariali medi, intesi come proxy del costo opportunità delle persone coinvolte, sorge il problema del differente (minore) costo del lavoro femminile, problema che, se trascurato, introduce elementi di artificiosità nell'analisi e, se considerato, introduce unbias nei risultati del- l'analisi stessa (gli interventi riguardanti le donne dovrebbero essere considerati con maggior favore di quelli che riguardano uomini, perché - a parità di risultato - sarebbero meno costosi e quindi darebbero luogo a valori $\mathrm{C} / \mathrm{E}$ più favorevoli).

\section{CEA e processo decisionale}

Ritenere che le decisioni allocative avvengano, nella realtà, sulla base soltanto delle liste di priorità ottenute ordinando per valore crescente $\mathrm{i}$ rapporti $\mathrm{C} / \mathrm{E}$ riferiti a un indicatore ampio di risultato come il QALY. E assumendo una prospettiva societaria, costituirebbe oggi un'imperdonabile ingenuità, comprensibile solo nei momenti eroici di fondazione della CEA (e delle altre tecniche di valutazione, economiche e non solo) ${ }^{\text {vii }}$. La politica, infatti, mantiene le sue ragioni, che non possono essere completamente catturate dalle variabili prese in considerazione dalla CEA: aspetti di equità e accessibilità ai servizi sanitari, benefici e costi esterni al settore sanitario ne rappresentano semplicemente esempi significativi.

Per quanto sia tecnicamente possibile utilizzare in modo meccanico i risultati della CEA e rappresentarli sotto forma di "classifiche", spesso risulta inappropriato farlo, in quanto la "CEA non è un processo decisionale completo" (17). Ciò non significa, naturalmente, escludere l'utilità pratica della CEA. Le informazioni che essa ha permesso di raccogliere ed elaborare possono svolgere un utile ruolo nel contribuire a orientare i decisori, non diversamente delle informazioni fornite ai consumatori dalle riviste che comparano pregi e difetti della maggioranza dei prodotti (beni o servizi) presenti sul mercato.

Non mancano inoltre $\mathrm{i}$ casi in cui una buona CEA è servita a capovolgere alcune credenze radicate, ad esempio che gli interventi di medicina preventiva siano sempre, per definizione, costo-efficaci ed ha contributo a ridimensionare alcuni programmi, inizialmente impostati con una frequenza di verifica "eccessiva" (dal punto di vista del costo-efficacia). Ne è esempio lo screening per il cancro alla cervice dell'utero, che è risultato determinare costi per vita salvata in crescita esponenziale al ridursi dell'intervallo tra un esame e l'altro (11): tale conoscenza ha contributo a rivedere, negli USA, la modellizzazione dei programmi nazionali di prevenzione.

In generale, si ritiene che il confronto diretto tra i rapporti $\mathrm{C} / \mathrm{E}$ possano avere un peso tanto maggiore nel processo decisionale quanto più simili sono gli interventi che si confrontano e più omogenee le popolazioni cui gli in-

\section{Nota vi}

Il caso di ponderazione più noto è quello operato dallo Stato dell'Oregon che, partendo da una valutazione dell'efficacia dei vari interventi sanitari, ha determinato l'elenco delle prestazioni cui sono ammessi gratuitamente $i$ beneficiari del programma di assicurazione pubblica. Oltre a quello dell' aggregazione, Daniels (5) individua altri tre problemi irrisolti, che contribuiscono a delineare la complessità dei temi che devono essere affrontati nel valutare i risultati dei programmi sanitari:

1) il problema del contrasto tra ricerca di accettabile equità distributiva e ricerca di risultati migliori: fino a quale grado dovrebbe essere favorita la produzione di risultati migliori piuttosto che cercare di fornire a tutti i pazienti l'opportunità di fruire delle risorse limitate? Fino a che punto investire in ricerca innovativa e diffondere nuove tecnologie ad alto costo, quando ancora molti soggetti non possono fruire di servizi e prestazioni consolidati ed efficaci a basso costo?

2) il problema delle priorità: quale grado di priorità dovremmo concedere al trattamento dei pazienti più gravi, ai pazienti disabili elo portatori di handicap, ai pazienti con minore aspettativa di vita, ai pazienti affetti da patologie croniche, ai pazienti terminali?

3) il problema della democrazia: possiamo usare un processo decisionale di tipo democratico per stabilire come allocare equamente le risorse o per giudicare se il razionamento ha prodotto risultati sanitari equi? (9). 


\section{Nota vii}

Per una lucida lettura della recente storia della valutazione delle politiche pubbliche nel Paese ove esse hanno visto il maggior sviluppo (gli USA) e una persuasiva periodizzazione della sua evoluzione si veda Stame (15). A un'iniziale (1960-

1975) atteggiamento ottimista verso la valutazione ha fatto seguito un atteggiamento pessimista (1975-1985), seguito poi da un atteggiamento più articolato e cauto (sono ora note le potenzialità, ma anche i limiti, della valutazione).

\section{Nota viii}

La restrizione è più forte che per gli altri tipi di ricerca originale, per $i$ quali è solo richiesto di dichiarare le eventuali sponsorizzazioni.

\section{Nota ix \\ La Food and Drug Administration ameri- cana ha emanato rego- le precise (nel docu- mento: 21 CFR Part 314,126) per le aziende farmaceutiche riguar- danti le modalità di di- mostrazione della co- sto-efficacia dei propri farmaci, soprattutto per quanto riguarda la comparazione con $i$ farmaci concorrenti (17).}

terventi sono somministrati. Nelle situazioni opposte, in cui la decisione coinvolge significative differenze nei trattamenti analizzati e nelle popolazioni di riferimento, i rapporti $\mathrm{C} / \mathrm{E}$ continuano a rappresentare informazioni essenziali, ma richiedono di essere valutate alla luce di circostanze e valori che non possono essere incorporati pienamente nell'analisi.

Infine, anche facendo uso di metodi di analisi fortemente standardizzati (per favorire la comparabilità dei risultati), vi sono situazioni nelle quali i rapporti $\mathrm{C} / \mathrm{E}$ non sono in grado di fornire utili termini di confronto relativamente a tutti gli interventi sanitari: ad esempio, il confronto diretto tra costo per QALY nel trattamento della schizofrenia e delle malattie cardiache, per la radicale differenza nei risultati, costituirà probabilmente solo uno dei fattori da considerare nella decisione, che dovrà essere assunta tuttavia sulla base di fattori non presenti nella CEA.

Usi correnti e potenziali della CEA: quali regole per quali utilizzi?

L'interesse verso le analisi CEA non sembra ridursi nel tempo ed è concentrato soprattutto nel settore dei farmaci. Notoriamente, questo sviluppo settoriale è favorito da molte circostanze, di natura sia tecnica (in primo luogo, la generale buona disponibilità di dati clinici sull'efficacia dei farmaci), sia politica (la pretesa restrizione nei finanziamenti pubblici disponibili per i sistemi sanitari, che spinge verso l'applicazione di regole value for money nei confronti dei nuovi farmaci, sono sempre più costosi di quelli che tendono a sostituire).

La spinta più consistente deriva peraltro dalla condizione di concorrenza monopolistica tra le grandi multinazionali farmaceutiche, che le induce a puntare con decisione sul vantaggio competitivo derivabile dalla (migliore) costo-efficacia dei propri prodotti: è questa situazione di mercato a mantenere sostenuta la loro domanda di studi CEA. Finora in due soli paesi (Australia e Ontario) le richieste delle aziende per far inserire i propri prodotti nei formulari nazionali dei farmaci ammessi al rimborso pubblico devono essere esplicitamente corredati da CEA. E i paesi dell'UE, pur limitandosi a richiedere alle aziende documentazione del fatto che i nuovi farmaci "valgano il prezzo richiesto", spingono anch'essi, seppure indirettamente, a sviluppare le CEA.

Insomma, è evidente che gli interessi economici in gioco erano e restano altissimi. Tanto da indurre gli editors di The New England Journal of Medicine ad assumere un atteggiamento rigorista nel definire i criteri di accettabilità di articoli originali su analisi co- sto-efficacia in tema di farmaci, ritenendo in tale modo di ridurre il rischio che conflitti di interesse abbiano ad influenzare le scelte metodologiche o a introdurre bias nella selezione dei dati oggetto di elaborazione: non vengono presi in considerazione gli articoli dei quali anche uno solo degli autori abbia una relazione finanziaria con una ditta sponsorizzatrice (13) viii.

Ciò spiega la forte spinta a regolamentare, in modo più o meno dettagliato, le modalità di redazione degli studi CEA.

A parte ogni altra considerazione, sembra utile invocare una ragione di buon senso a operare in questa direzione, come quella espressa dagli autori del più volte citato Rapporto dell'US Department of Health and Human Services (17): "Differences in reported health outcomes, costs, and cost-effectiveness ratios should reflect, as much as practicably possible, true differences in the consequences of the interventions and not be artifacts introduced by unnecessary differences in method".

In questo contesto, ha senso porsi almeno due domande, simili ma distinte.

La prima: è auspicabile, mediante la formulazione di dettagliate indicazioni metodologiche, vincolare i contenuti delle CEA utilizzate nei processi decisionali pubblici (a fini di regolamentazione)?

La seconda: sono maturi i tempi per una rigorosa standardizzazione della ricerca in ambito CEA?

A parere di chi scrive, le due domande richiedono risposte differenziate.

La prima domanda si riferisce ai possibili utilizzi diretti dei risultati CEA, a fini di regolamentazione pubblica: le decisioni, cioè, (a livello di sistema Paese e/o di sistema sanitario) di ammettere un nuovo farmaco e/o di determinarne il prezzo di vendita e/o di inserirlo/non inserirlo nell' elenco dei farmaci acquistabili/rimborsabili dall'organizzazione sanitaria pubblica (quest'ultima decisione può riguardare, con riferimento al SSN italiano, il livello nazionale, quello regionale e della singola azienda sanitaria, locale oppure ospedaliera). Come sembrano indicare l'esperienza australiana (1) e quella canadese (4) e quella americana della Food and Drug Administration (peraltro più limitata ${ }^{\mathrm{ix}}$ ) pare difficile negare l'esistenza di prerequisiti sufficienti a fondare un insieme di regole vincolanti per le CEA, sottomesse a fini autorizzativi ad agenzie pubbliche (2).

Se ciò fosse vero, perché non si è assistito a una generalizzazione di tali prassi?

Una risposta schematica sottolinea il variegato potere di persuasione delle multinazionali farmaceutiche, che temerebbero l'im- 
patto di analisi CEA condotte con criteri vincolanti sul comportamento delle autorità sanitarie nazionali, che potrebbero condurre, al limite, a selezionare per l'immissione sul mercato i soli farmaci con i migliori rapporti $\mathrm{C} / \mathrm{E}$.

Una seconda risposta, più ponderata, senza negare totale fondamento alla considerazione che precede, l'affiancherebbe con altri riferimenti, che possono indurre a una valutazione più sfumata e meno cinica.

Mi limito a pochi accenni, ciascuno dei quali meriterebbe ampi sviluppi.

Un primo fattore esplicativo è di ordine economico generale e richiama l'opportunità, nell'interesse pubblico (in una lettura di medio e lungo periodo), che gli investimenti delle imprese farmaceutiche in ricerca e sviluppo mantengano nel tempo livelli sufficientemente elevati, come è consentito da livelli ritenuti "accettabili" non solo degli utili sulla produzione corrente, ma anche del livello di incertezza sulla possibilità che i nuovi prodotti siano immessi convenientemente sul mercato.

Un secondo fattore è di natura tecnica e culturale: come la storia del progresso scientifico e tecnologico insegna, spesso si impara dagli errori passati e ci vuole tempo affinché siano riconoscibili tutti gli effetti dei farmaci (positivi e negativi) sulla salute umana. Le nostre società hanno smarrito l'arte dell'attesa: si pretendono decisioni rapide, non si deve "fermare il progresso della scienza". Da ciò discende che l'orizzonte temporale delle CEA, quali di fatto sono praticate, tende a essere più di breve che di lungo periodo ed è quindi coerente più con una valutazione dell' efficacy che della vera effectiveness dei trattamenti che vuole comparare. La consapevolezza del contrasto tra i tempi della scienza e i tempi della decisione può spiegare la permanente riluttanza ad assumere come vincolanti, nei processi decisionali collettivi, i risultati di analisi che, per quanto ci si sforzi di superane i limiti, sono tuttora incomplete e riferite a un orizzonte temporale e spaziale che si percepisce come troppo limitato.

Un terzo fattore riguarda i limiti del ruolo che la valutazione economica può legittimamente rivendicare nei processi di decisione collettiva. Come si è cercato di rappresentare nel paragrafo precedente, la CEA non è oggi in grado di soddisfare (lo sarà mai?) tutte le esigenze e rispondere persuasivamente a tutte le domande che i responsabili politici legittimamente intendono tenere presenti nel decidere. Da questo punto di vista, voler forzare l'utilizzo della CEA fino a renderne assolutamente vincolanti i risultati potrebbe essere interpretato come un'indebita invasione del campo proprio della politica.

La risposta alla seconda domanda (se sia- no maturi i tempi per una rigorosa standardizzazione della ricerca in ambito CEA) $\mathrm{mi}$ pare possa essere: solo parzialmente.

Da un lato, infatti, si deve riconoscere che è venuta crescendo l'area di consenso scientifico sul disegno e sui contenuti metodologici e di merito dell'analisi. Fornisco qualche indizio in questo senso:

? uno degli economisti sanitari più noti in ambito europeo, l'inglese Mike Drummond, in due occasioni ha specificato, sotto forma di check-list, le domande cui una "buona" analisi farmacoeconomica (sostanzialmente di tipo CEA) deve sapere rispondere. La prima checklist, del 1987 (6, 8, 20) comprendeva 10 punti, che sono aumentati a 35 nella griglia proposta nove anni più tardi $(7,3)$, estesa ad aspetti prima trascurati;

? il rapporto finale redatto dal gruppo di esperti radunati dal Ministero della Sanità americano, al termine di oltre due anni di lavoro (17) comprende, in conclusione di otto dei nove capitoli tematici, altrettanti insiemi di raccomandazioni sulle caratteristiche che una buona CEA dovrebbe possedere e sui temi che richiedono invece ulteriore ricerca (perché su di essi non si è potuta realizzare unanimità di consensi nel gruppo di lavoro). Una semplice notazione quantitativa: il rapporto formula 76 raccomandazioni "positive" e individua 10 aree di dissenso, che richiedono ulteriore ricerca.

D'altro lato, il consenso si manifesta in raccomandazioni di carattere piuttosto generale, non diverse da quelle proprie di ogni attività scientifica (che traducono in comandamenti dettagliati le regole generali della coerenza, della trasparenza e della replicabilità), seppure arricchite da un numero crescente di suggerimenti dettati dall'esperienza.

$\mathrm{Su}$ alcuni punti cruciali dell'analisi prevale la consapevolezza che sarebbe estremamente pericoloso per gli sviluppi ulteriori della ricerca privilegiare sempre linee di condotta, metodi di indagine e scelta di parametri rigidi (ad esempio, sul tema centrale della misurazione del risultato e della modellizzazione dell'incertezza) (17). Sintomatico di questo atteggiamento complessivo è, nel rapporto americano, la proposta, formulata al fine di garantire elevata comparabilità tra i risultati di CEA condotte in ambiti diversi, di sostituire un insieme vincolante di regole con un reference case, definito "a standard set of methodologic practices that an analyst would seek to follow in a CE study"x.

La funzione assegnata al reference case è di costituire una sorta di duale (o termine di riferimento standard) per le ricerche che si allontanassero, per assunzioni formulate e me-

\section{Nota $\mathbf{x}$}

Le due appendici finali del rapporto presentano esempi di reference case completamente elaborati relativi, il primo, alla valutazione comparativa di strategie per prevenire i difetti al tubo neurale, il secondo, alle terapie farmacologiche $e$ dietologiche alla riduzione del colesterolo nel sangue degli adulti. 
todi praticati, dalle raccomandazioni espresse nel rapporto.

La possibilità di agire in difformità dalle "raccomandazioni" è quindi prevista e legittimata, anzi viene incoraggiata in vista degli utilizzi particolari cui le analisi possono, correttamente, tendere. E ciò sembra convergere con un analogo atteggiamento di fondo cui è pervenuta la valutazione delle politiche pubbliche, che ritiene il pluralismo dei metodi e l'attenzione alle legittime aspettative dei destinatari della valutazione la nuova ortodossia (15).

D'altra parte, un apprezzabile grado di comparabilità (nei limiti in cui ha senso comparare), tra i risultati di CEA condotte rispetto a interventi di carattere estremamente eterogeneo (dalle norme di sicurezza ai farmaci, al controllo degli inquinanti) è ottenibile anche ex-post, purché le indagini originali siano state condotte secondo regole condivise dalla comunità scientifica (16).

\section{BIBLIOGRAFIA}

1. Australia Commomwealth Department of Health, Housing and Community Services (ACDHHCS): Guidelines for the pharmaceutical industry of submission to the Pharmaceutical Benefits Advisory Committee, Commonwealth Department, Canberra, 1992

2. Barosi M., Garattini L.: Confronto internazionale delle "guidelines" in farmacoeconomia, FarmEconomia, 1996, 2: $5-14$

3. Bondonio P.: Tempo di linee guida per gli autori di FarmEconomia, FarmEconomia, 1996, 4: 5-10

4. Canadian Coordinating Office on Health Technology Assessment (CCOHT): Guidelines for economic evaluation of pharmaceuticals, CCOHTA, Ottawa, 1994Cresa: Aspetti economici della demenza senile, Angeli, Milano, 1993

5. Daniels N.: Four unresolved rationing problems: a challenge, Hastings Cent Rep, 1994, 24: 27-29

6. Drummond M.F., Stoddart G., Torrance G.: Methods for the economic evaluation of health care programmes, Oxford University Press, Oxford, 1987 (trad. it. Metodi per la valutazione economica dei programmi sanitari, a cura di V. Ghetti, Angeli, Milano, 1993).

7. Drummond M. F., Jefferson T.O. (on behalf of the BMJ Economic Evaluation Working Party): Guidelines for authors and peer reviewers of economic submissions to the BMJ, BMJ, 1996, 313: 275-283

8. Eandi M.: Una checklist per analizzare la qualità di una valutazione farmacoeconomica, FarmEconomia, 1994, 1: 16-17

9. Eandi M., Bondonio P., Principi di farmacoeconomia, I/O n.1, Medicom editore, Milano, 1999 (in corso di stampa)

10. Eandi M., Bondonio P., Dirindin N.: Farmacoeconomia e terapia antibiotica, A.B.E. Edizioni scientifiche, Milano, 1993.

11. Eddy D.M.: Screening for cervical cancer, Ann. Intern. Med., 1990, 113: 214-226

12. Greenberg P.E., Stiglin L.E., Finkelstein S.N., Berndt E.R.: Depression: a neglected major illness, J Clin Psychiatry, Nov.1993, 54: 419-24.

13. Kassirer J.P., Angell M.: The journal's policy on cost-effectiveness analysis, N Engl J Med, 1994, 331: 912-16

14. Rawls J.: A theory of justice, Harvard University Press, Cambridge, MA, 1971 (trad. it.: Teoria della giustizia, Feltrinelli, Milano, 1982)

15. Stame N.: L'esperienza della valutazione, Edizioni SEAM, Roma, 1998

16. Tengs T.O et al.: Five-hundred life-saving interventions and their cost-effectiveness, Risk Analysis, 1995, 15: 369-390.

17. U.S. Department of Health and Human Services, Public Health Service, Cost-effectiveness in health and medicine, Report to the U.S. Public Health Service by the Panel on cost-effectiveness in health and medicine, Washington, D.C., 1996.

18. Weinstein M.C., Stason W.B., Foundations of cost-effectiveness analysis for health and medical practices, N Engl J Med, 1977, 296: 716-21.

19. Zara G.P.: Aggiornamento in tema di “costo di malattia”, FarmEconomia, 1996, 1: 40-45

20. Zara G.P.: Rapporto costo-efficacia del trattamento precoce con AZT in soggetti HIV-positivi, FarmEconomia, 1994, 1: 22-24 